\title{
Sudden death in Lesch-Nyhan disease
}

\author{
Vladimir Kostadinov Neychev, MD and H A Jinnah, MD, PhD ${ }^{*}$ \\ Department of Neurology, Johns Hopkins Hospital, Baltimore, MD, USA
}

\begin{abstract}
To increase awareness of sudden and unexpected death in Lesch-Nyhan disease (LND) and to explore its potential causes, we report the anteceding clinical features and laboratory evaluations of five males with LND who ultimately experienced sudden and unexpected death, along with three additional males who suffered serious respiratory events during life. The ages of patients ranged from 2 to 45 years. The cause of sudden death in LND appears to have a respiratory rather than a cardiogenic basis. All cases cannot be linked readily with a single respiratory process. Instead, different respiratory processes appear to operate in different cases. These may include aspiration, laryngospasm, central apnea, cyanotic breath-holding spells, and high cervical spine damage. Better recognition of these processes will help to guide appropriate workup and management that could include chest imaging, endoscopy of the airways, polysomnography, electroencephalogram, and brain and/or spine imaging.
\end{abstract}

Lesch-Nyhan disease (LND) is an X-linked recessive disorder resulting from hypoxanthineguanine phosphoribosyl transferase (HPRT) deficiency. It was recognized and established as a nosological entity in $1964 .{ }^{1}$ Since then, it has been well studied clinically, biochemically, and genetically. ${ }^{2-9}$ When fully developed the clinical picture of the disease is represented by three major elements including overproduction of uric acid, neurological disability, and characteristic behavioral problems. The usual neurological signs associated with LND are of extrapyramidal origin. Dystonia is universal, and often combined with choreoathetosis, ballismus, and signs of pyramidal tract dysfunction such as spasticity and hyperreflexia. ${ }^{5}$ Hallmarks of the behavioral syndrome include severe self-injury, aggressive behaviors, and disturbing interpersonal behaviors. ${ }^{9}$

Shortly after its initial recognition, the causes of death among most patients with this condition were known to include aspiration pneumonia, malnutrition due to severe dysphagia, or chronic nephrolithiasis with renal failure or urosepsis. Fortunately, modern medical management has reduced these complications to a minimum. Complications from nephrolithiasis can usually be controlled with allopurinol to inhibit the production of uric acid. Malnutrition and aspiration pneumonia can be managed by tailoring dietary habits to swallowing abilities, by using parenteral nutrition or gastrostomy tubes, and using antibiotics when needed. As a result of improvements in medical management, these patients are often now surviving beyond their third decade of life. The lengthier survivals have led to increasing recognition that a significant proportion of them may die suddenly and unexpectedly from unknown causes.

The cause of sudden death is difficult to evaluate after it has occurred. Detailed autopsy studies of some cases have failed to reveal an explanation. ${ }^{7,8}$ Here, we report eight cases with life-threatening respiratory events, including five who ultimately died. Potential

*Correspondence to second author at Meyer Room 6-181, Department of Neurology, Johns Hopkins University, Baltimore, MD 21287, USA.hjinnah@jhmi.edu. 
reasons for sudden death are considered, with suggestions for causes and clinical management.

\section{Case reports}

Case 1 was noted to have motor delay by 2 to 3 months of age, and generalized dystonia was apparent by 8 months of age. He carried a diagnosis of cerebral palsy (CP) until 7 years of age, when the development of recurrent finger biting led to the suspicion of LND. Hyperuricemia was never apparent, and the diagnosis was established by reduced HPRT enzyme activity in erythrocytes.

He was first evaluated by us at age 15 years. By this time, he had developed many of the problems commonly seen in LND including severe generalized dystonia, dysarthria, dysphagia with frequent emesis, cognitive impairment, and multiple forms of self-injury. Additionally, he had experienced three episodes of respiratory arrest with coma beginning at 9 years of age, usually after an episode of emesis. He presented with his fourth event, which began with emesis, followed 3 to 5 minutes later by respiratory arrest. He then developed bradycardia, hypotension, and obtundation.

Aspiration of vomited gastric material with subsequent airway obstruction was considered the most probable reason for respiratory arrest, with hypoxia leading to secondary cardiac dysfunction, because the patient had a history of emesis always preceding his breathing arrests. However, nasolaryngopharyngoscopy during the last crisis failed to provide any evidence for aspiration with airway obstruction. He eventually recovered and a cause for his respiratory arrest was not found, but he died unexpectedly and suddenly in his sleep at the age of 15 years.

Case 2 presented to us at age 37 after an episode of unexplained respiratory arrest. His early developmental history was not available, but his diagnosis was established by molecular and biochemical methods. He had a G580A mutation in the hprt gene resulting in a glycine to alanine substitution and non-detectable enzyme activity. He displayed scarring of the face and hands from self-injury, cognitive impairment, severe generalized dystonia with spasticity, dysarthria and dysphagia, gastroesophageal reflux, nephrolithiasis, and macrocytic anemia.

The initial concern again was for aspiration with subsequent arrest from sepsis or hypoxia; however, nasopharyngoscopy revealed no structural obstruction to airflow, computed tomography (CT) of the chest was unrevealing, and blood cultures failed to grow any organisms. Electrocardiogram revealed a stable right bundle branch block and cardiac monitoring for 24 hours revealed no abnormality. Cardiology consultation provided no suspicion for a primary cardiac event, and vascular studies of the lower limb were negative for thrombosis.

His history of violent retrocollis and examination features, suggestive of cervical myelopathy, led to concern for a high cervical-spine injury leading to dysfunction of respiratory motor neurons in the cervical cord. Magnetic resonance imaging (MRI) of the cervical spine showed a globally narrowed cord, beginning just caudal to the brainstem where it was not likely to suffer mechanical injury. He also had advanced degenerative changes, with severe canal stenosis and foraminal narrowing at C5-C6. These changes were deemed unlikely causes for respiratory compromise because they occurred at a level that does not typically affect respiratory motor neurons of the cervical cord.

Dysfunction of the brainstem with central apnea was also suspected as a possible cause, as his sleep study was abnormal with complete absence of stages 3 to 4 . However, no apnea or 
significant oxygen desaturation was found to support central apnea as a primary cause. The cause for his breathing arrest was not established, though there was no further cardiac or pulmonary event until he died suddenly and unexpectedly at the age of 45 years.

Case 3 was noted to have motor delay by 4 months of age and had developed dystonic posturing by 12 months of age. He carried a diagnosis of $\mathrm{CP}$ until he developed urinary obstruction due to uric acid stones. Genetic studies revealed a splicing mutation (IVS7+5G>A) in the hprt gene predicting exclusion of exon 7 and null enzyme function.

By the time of his evaluation at age 6 years, he had developed many of the problems seen in LND, except for self-injurious behavior. He had severe generalized dystonia. He was cognitively impaired and non-verbal. He had minor dysphagia but reflux and emesis were not apparent. He also had recurrent spells of apnea leading to cyanosis and obtundation beginning at 5 years. The spells occurred many times per hour, each lasting 1 to 5 minutes. They were more common when he was excited or under stress. Many of the spells were observed to follow a period of hyperventilation. They were not associated with stridor or signs of aspiration or respiratory distress.

Two extended video-electroencephalogram (EEG) studies revealed recurrent apnea with oxygen desaturations below 50\%, followed by diffuse slowing on the EEG. The EEG changes were attributed to hypoxia rather than seizures and the spells did not respond to five different anticonvulsants (phenobarbital, carbamazepine, levetiracetam, diazepam, gabapentin) or caffeine. During sleep he continued to have episodes of central apnea, but they were less frequent and less severe. With 363 minutes of sleep he had 32 apneas up to 21 seconds long, with a maximum oxygen desaturation of $88 \%$. Electrocardiography and echocardiography were normal. MRI of the brain and cervical spine were not revealing.

Because excitement and hyperventilation often preceded his apneas, breath-holding spells or an extreme form of post-hyperventilation apnea were considered. The severe episodes of central expiratory apnea after hyperventilation that were witnessed in this patient, and attenuation of the spells during sleep, when he did not have anxiety-related hyperventilation, could support either possibility. He continued to have apneic spells at age 8, though their frequency and severity have been reduced by efforts directed to the management of psychological factors leading to hyperventilation.

Case 4 was born by emergency caesarean section after identification of cardiac decelerations. Motor delay was noted at 4 months of age, and dystonic posturing became increasingly apparent at 6 to 12 months of age. He carried a diagnosis of CP until he began to bite his thumb at 23 months of age. The serum uric acid was found to be increased to $9 \mathrm{mg} / \mathrm{dL}$, and his diagnosis was established by molecular methods, which demonstrated 10del1 in the hprt gene, predicting null enzyme function.

In addition to the typical problems observed in young patients with LND, he had severe and recurrent gastroesophageal reflux leading to Nissen fundoplication with a gastrostomy tube. One month after surgery, he developed recurrent apnea with cyanosis. The apnea often appeared after a period of agitation with loud crying. It was sometimes associated with loss of consciousness for up to 15 seconds.

His workup including brain CT, chest, and neck radiography, and echocardiography was generally unremarkable. Cyanotic breath-holding spells were considered as a possible explanation for his breathing problem; however, the episodes of apnea spontaneously remitted before any definite cause could be found. 
Case 5 was first noted to have difficulty with motor function at the age of 7 months with hypotonia. Dystonic posturing and self-injury became increasingly apparent at 3 to 4 years of age. He carried a diagnosis of CP until 8 years of age when the development of uric acid crystalluria led to suspicions of LND. This was confirmed by genetic studies that revealed a missense mutation (A611G) in the hprt gene leading to substitution of arginine for histidine at codon 204.

By the time of his evaluation at age 8 years, he had undergone Nissen fundoplication with gastrostomy tube due to hiatal hernia with severe dysphagia. A jejunostomy tube and laparotomy for ileus mechanicus were later required. After surgery he developed episodes of labored and deep breathing when he lay down at night. However, he has not had any witnessed episodes of apnea, cyanosis, or unexplained obtundation.

His chest X-ray revealed a large recurrent paraesophageal Nissen hernia that enlarged and occupied the mid-lower chest compartment when lying down, a problem that could provoke respiratory compromise.

Case 6 had classic LND but was not evaluated by us during life. His records were reviewed after sudden death at the age of 41 years. Autopsy revealed disfiguration of the lower lip consistent with self-mutilation, bilateral stones in the kidneys and ureters from uric acid overproduction, as well as scoliosis and unilateral neck muscle hypertrophy from generalized dystonia. There was no evidence for a cardiac event. No obstructions were found in the tracheobronchial tree. A cause for sudden death was still not apparent after the autopsy, though information from the brain and spinal cord was not available.

Case 7 had classic LND, but was not evaluated by us during life. With no anteceding medical problem, he was found apneic and pulseless with no apparent cause. He was successfully resuscitated, but died shortly after at the age of 34 years. At autopsy, several findings confirmed LND including numerous small abrasions over the posterior aspects of the scalp and on the lips, absence of the right thumb, and bilateral nephrolithiasis. A thorough cardiopulmonary evaluation at autopsy revealed no apparent cause for his death, though information from the brain and spinal cord was not available.

Case 8 was not evaluated by us during life. He suffered respiratory arrest with coma in his home, which required intubation and ventilation. He remained comatose with no evidence of brainstem function until he died at the age of 21 years. He was well before the arrest, with no obvious cause for the arrest. Autopsy was not performed, and the cause of his sudden death was not determined.

\section{Discussion}

Modern medical tools have markedly improved the outlook of patients with LND. Their increased longevity has made it increasingly clear that they may die suddenly and unexpectedly without an obvious cause. The reasons for sudden death remain unclear, because the events immediately preceding death are difficult to determine when death occurs with little or no prior warning. Though a formal study of this phenomenon is not feasible because of the rarity of LND combined with the logistical difficulties inherent in sudden death, the current series of cases is valuable for drawing attention to the problem and for beginning to develop a better understanding of its potential causes. Potential causes suggested by the current series include unrecognized aspiration, laryngospasm, central apnea, cervical spine damage, cyanotic breath-holding, a behavioral process, pulmonary embolus, or a cardiac event. The available evidence provides little support for a primary cardiac process in LND; instead, it seems to point toward respiratory events with subsequent cardiac arrest (Table I). 
Aspiration with obstruction of the airways by gastric contents may seem one of the most likely causes, especially considering the high frequency of recurrent emesis in this disorder. Though some of the current cases ( 1 and 2 ) provided histories of emesis before respiratory arrest, most had extensive evaluations that provided no evidence for aspiration causing respiratory arrest or sudden death. In other cases (case 3), recurrent apneas were witnessed with no suggestion of prior emesis or aspiration as in prior reports. ${ }^{7,8}$ Based on these observations, it seems that aspiration with airway obstruction may occur, but it provides an inadequate explanation for all cases.

Laryngospasm provides a related explanation. Emesis or aspiration with irritation of the larynx or pharynx can trigger laryngospasm, or laryngeal dystonia, which often resolves after loss of consciousness. ${ }^{10,11}$ As a result, this process could be difficult to detect after it has occurred. Because patients with LND exhibit severe generalized dystonia with involvement of almost all parts of the body, it is likely that they are at increased risk for laryngeal dystonia. Laryngeal breathing dystonia has been reported to cause airway obstruction in other dystonic disorders. ${ }^{12-14}$ This mechanism should be considered in all cases, as it can be treated with local injection of botulinum toxin.

Another explanation for sudden death involves dysfunction of brainstem respiratory centers. Instability of the respiratory oscillator in the medulla oblongata has been implicated in respiratory dysrhythmias and sudden deaths observed in other disorders such as Rett syndrome. ${ }^{15}$ This mechanism has been considered in detail in previous studies of patients with $\mathrm{LND},{ }^{7,16,17}$ though evidence has so far been lacking. The sleep studies of cases 2 and 3 provided evidence for a defect in central respiratory control similar to previous sleep studies, ${ }^{7,18}$ though it was difficult to conclude that it was the sole process responsible for respiratory dysfunction and it did not appear to operate in other cases.

A related explanation involves centrally mediated expiratory apnea, also known as cyanotic breath-holding spells. ${ }^{19,20}$ A mortality of $15.7 \%$ was reported in a study of 51 infants and young children with cyanotic breath-holding. ${ }^{21}$ Impaired interactions between brainstem control of respiration and central sympathetic activity have been implicated in this disorder. ${ }^{21-23}$ In this regard, a study revealing that under conditions of emotional or postural stress the plasma concentrations of norepinephrine in patients with LND increased less than in a normal population ${ }^{24}$ suggests the possibility of impaired central sympathetic control of respiration in LND.

High cervical-spine injury with subsequent dysfunction of respiratory motor neurons in the cervical cord at C3-C4 could also produce respiratory dysfunction. Patients with LND are at increased risk for injury to the cervical spine, because of their severe cervical dystonia and frequent sudden retrocollis, leading to degenerative changes of the cervical spine and spinal instability. ${ }^{5,25}$ Cervical imaging in two of our cases provided evidence for degenerative changes of the spine, but these were not sufficient to provoke respiratory compromise.

In case 3, among the suspicions for recurrent apnea was a behavioral process such as spells of voluntary breath-holding. This possibility must be considered, because difficult behaviors, particularly those that lead to heightened concern and attention, are well known in LND. The breath-holding that occurs with cyanotic breath-holding is involuntary and reflexive, occurring after full expiration, in contrast to volitional breath-holding, which follows prolonged inspiration. ${ }^{20}$ The occurrence of apnea after expiration and the sleep studies documenting a central respiratory defect reduced our enthusiasm for a purely behavioral process accounted for his apneas, though contributions from a behavioral process could not be excluded for the more severe daytime events. 
In summary, patients with LND may succumb to sudden death, despite optimal medical management. An exploration of potential causes does not point to a single causative process for all cases. There seems little evidence for a primary cardiac process, though respiratory problems are evident. When life-threatening breathing events occur, several investigations tailed to the clinical features are warranted including chest imaging, endoscopy of the airways, polysomnography, EEG, and imaging of the brain and/or spine. Further observations in additional cases will be required to develop a better understanding of the cause(s) for respiratory dysfunction in LND so that better management strategies for sudden death can be developed.

\section{References}

1. Lesch M, Nyhan WL. A familial disorder of uric acid metabolism and central nervous system function. Am J Med. 1964; 36:561-570. [PubMed: 14142409]

2. Jinnah HA, Harris JC, Nyhan WL, O’Neill JP. The spectrum of mutations causing HPRT deficiency: an update. Nucleosides Nucleotides Nucleic Acids. 2004; 23:1153-1160. [PubMed: 15571220]

3. Visser JE, Bar PR, Jinnah HA. Lesch-Nyhan disease and the basal ganglia. Brain Res Brain Res Rev. 2000; 32:449-475. [PubMed: 10760551]

4. Schretlen DJ, Harris JC, Park KS, Jinnah HA, del Pozo NO. Neurocognitive functioning in LeschNyhan disease and partial hypoxanthine-guanine phosphoribosyltransferase deficiency. J Int Neuropsychol Soc. 2001; 7:805-812. [PubMed: 11771623]

5. Jinnah HA, Visser JE, Harris JC, Verdu A, Larovere L, Cenallos-Picot I, Gonzalez-Alegre P, Neychev V, Torres RJ, Dulac O, et al. Delineation of the motor disorder of Lesch-Nyhan disease. Brain. 2006; 129:1201-1217. [PubMed: 16549399]

6. Christie R, Bay C, Kaufman IA, Bakay B, Borden M, Nyhan WL. Lesch-Nyhan disease: clinical experience with nineteen patients. Dev Med Child Neurol. 1982; 24:293-306. [PubMed: 7095300]

7. Watts RW, Spellacy E, Gibbs DA, Allsop J, McKeran RO, Slavin GE. Clinical, post-mortem, biochemical and therapeutic observations on the Lesch-Nyhan syndrome with particular reference to the neurological manifestations. Q J Med. 1982; 201:43-78. [PubMed: 7111674]

8. Mizuno T. Long-term follow-up of ten patients with Lesch-Nyhan syndrome. Neuropediatrics. 1986; 17:158-161. [PubMed: 3762872]

9. Schretlen DJ, Ward J, Meyer SM, Yun J, Puig JG, Nyhan WL, Jinnah HA, Harris JC. Behavioral aspects of Lesch-Nyhan disease and its variants. Dev Med Child Neurol. 2005; 47:673-677. [PubMed: 16174310]

10. Morrison M, Rammage L, Emami AJ. The irritable larynx syndrome. J Voice. 1999; 13:447-455. [PubMed: 10498060]

11. Grillone GA, Chan T. Laryngeal dystonia. Otolaryngol Clin North Am. 2006; 39:87-100. [PubMed: 16469657]

12. Gdynia HJ, Kassubek J, Sperfeld AD. Laryngospasm in neurological diseases. Neurocrit Care. 2006; 4:163-167. [PubMed: 16627908]

13. Zwirner P, Dressler D, Kruse E. Spasmodic laryngeal dyspnea: a rare manifestation of laryngeal dystonia. Eur Arch Otorhinolaryngol. 1997; 254:242-245. [PubMed: 9195149]

14. Blitzer A, Brin MF. Laryngeal dystonia: a series with botulinum toxin therapy. Ann Otol Rhinol Laryngol. 1991; 100:85-89. [PubMed: 1992905]

15. Julu PO, Kerr AM, Hansen S, Apartopoulos F, Jamal GA. Immaturity of medullary cardiorespiratory neurones leading to inappropriate autonomic reactions as a likely cause of sudden death in Rett's syndrome. Arch Dis Child. 1997; 77:464-465. [PubMed: 9487980]

16. McCreanor GM, Harkness RA. Lesch-Nyhan syndrome and its pathogenesis: normal nicotinamideadenine dinucleotide but reduced ATP concentrations that correlate with reduced poly(ADPribose) synthetase activity in HPRT-deficient lymphoblasts. J Inherit Metab Dis. 1995; 18:737747. [PubMed: 8750613]

17. Lynch BJ, Noetzel MJ. Recurrent coma and Lesch-Nyhan syndrome. Pediatr Neurol. 1991; 7:389_ 391. [PubMed: 1764145] 
18. Saito Y, Hanaoka S, Fukumizu M, Morita H, Ogawa T, Takahashi K, Ito M, Hashimoto T. Polysomnographic studies of Lesch-Nyhan syndrome. Brain Dev. 1998; 20:579-585. [PubMed: 9865540]

19. Fujisawa H, Yoshida Y, Niida Y, Hasegawa M, Yamashita J. Cyanotic breath-holding spell: a lifethreatening complication after radical resection of a cervicomedullary ganglioglioma. Pediatr Neurosurg. 2005; 41:93-97. [PubMed: 15942280]

20. Breningstall GN. Breath-holding spells. Pediatr Neurol. 1996; 14:91-97. [PubMed: 8703234]

21. Southall DP, Samuels MP, Talbert DG. Recurrent cyanotic episodes with severe arterial hypoxaemia and intrapulmonary shunting: a mechanism for sudden death. Arch Dis Child. 1990; 65:953-961. [PubMed: 2221968]

22. Kohyama J, Hasegawa T, Shimohira M, Fukumizu M, Iwakawa Y. Rapid eye movement sleep in breath holders. J Child Neurol. 2000; 15:449-452. [PubMed: 10921515]

23. Kahn A, Rebuffat E, Sottiaux M, Muller MF, Bochner A, Grosswasser J. Brief airway obstruction during sleep in infants with BHS. J Pediatr. 1990; 117:188-193. [PubMed: 2380815]

24. Lake CR, Ziegler MG. Lesch-Nyhan syndrome: low dopamine-beta-hydroxylase activity and diminished sympathetic response to stress and posture. Science. 1977; 196:905-906. [PubMed: 860124]

25. Shewell PC, Thompson AG. Atlantoaxial instability in Lesch-Nyhan syndrome. Spine. 1996; 21:757-762. [PubMed: 8882700] 


\section{Table I}

Characteristics of cases in this study

\begin{tabular}{lrrr}
\hline Case & Age at onset of apnea & Clinical outcome & Suspected cause of apnea \\
\hline 1 & $9 y$ & Sudden death at 15y & Laryngospasm or central apnea \\
2 & $37 y$ & Sudden death at 45y & Laryngospasm or central apnea \\
3 & $5 y$ & Persistent apneas at 9y & Central apnea and/or behavioral \\
4 & $18 \mathrm{mo}$ & Spontaneous resolution by 2y & Laryngospasm or mechanical (GI) \\
5 & $8 \mathrm{y}$ & Spontaneous resolution by 13y & Mechanical (GI) \\
6 & NA & Sudden death at age 41y & Undetermined after autopsy \\
7 & NA & Sudden death at age 34y & Undetermined after autopsy \\
8 & NA & Sudden death at age 21y & Undetermined after autopsy \\
\hline
\end{tabular}

GI, gastrointestinal; NA, not available. 